\title{
Imaging of metastatic cervical nodes: is CT helpful in differentiation of squamous cell carcinoma (SCC) from non-SCC groups?
}

\author{
Lamya A. Eissa ${ }^{1 *}$ and Ahmed Mohamed Mehanna ${ }^{2}$
}

\begin{abstract}
Background: Cervical node metastasis is frequently encountered in $\mathrm{CT}$ neck of patients with squamous cell carcinoma (SCC) and non-(SCC). Differentiation between both entities carries its value on prognosis and choice of treatment plans. The purpose of the study was to compare between the SCC and non-SCC metastatic cervical adenopathies according to different imaging parameters with the use of comparative statistical analysis of any of these criteria. This was made by retrospectively studying 157 patients. Imaging analyzed the following parameters: nodal size, laterality, nodal levels, "grouping" of nodes, nodal shape, and "periphery," as well as the presence or absence of nodal "necrosis." Statistics are made to show significant differences between both groups.

Results: The criterion of necrosis had statistical significance, being more prevalent among the SCC groups. Involvement of levels I, II, and supra clavicular was more prevalent among SCC patients. Age and sex had also some statistical significance.

Conclusions: The combination of different imaging parameters could distinguish SCC from non-SCC. Nodal "necrosis" — (excluding "cystic") — combined with involvement of nodal levels II and being in older-aged men were statistically significant in the SCC compared to non-SCC.
\end{abstract}

Keywords: Node, CT, SCC, Metastasis

\section{Background}

Cervical lymph node metastasis is frequently encountered in CT neck examinations of patients with squamous cell carcinoma (SCC) and non-squamous cell carcinoma (non-SCC). The presence of lymph node metastasis is considered as an adverse prognostic factor in patients with head and neck cancers [1]. Dunne et al. [2] showed a near 5-year survival rates between 17 and $55.8 \%$ for SCCs with cervical node metastases compared with others between 44.6 and $76 \%$ for SCC patients without cervical node metastases [2].

Accurate identification of cervical lymph node metastases is essential for choosing the most appropriate

\footnotetext{
* Correspondence: lamya.eissa@gmail.com

${ }^{1}$ Department of Diagnostic and Interventional Radiology, Alexandria Faculty of Medicine, Alexandria 21131, Egypt

Full list of author information is available at the end of the article
}

treatment [2-4]. Delineation of cervical nodal metastasis is more accurately performed with imaging than with clinical palpation; imaging is widely used in both pre-treatment staging and detection of nodal recurrences.

Cross-sectional imaging, including computed tomography $(\mathrm{CT})$ and magnetic resonance imaging (MRI), is routinely performed to assess the primary tumor and to examine for lymph nodes along the drainage areas of the tumor. Other imaging modalities currently used are ultrasound (US). Recently, some novel imaging techniques have been developed in attempts to improve the accuracy of the detection of nodal metastasis [3-5].

Multiple cross-sectional criteria have been developed to diagnose metastatic disease [5]. Nodes (JD) are considered to be metastatic if central necrosis or extracapsular spread is present irrespective of size, or if the

\section{Springer Open}

(ㅇ The Author(s). 2020 Open Access This article is licensed under a Creative Commons Attribution 4.0 International License, which permits use, sharing, adaptation, distribution and reproduction in any medium or format, as long as you give appropriate credit to the original author(s) and the source, provide a link to the Creative Commons licence, and indicate if changes were made. The images or other third party material in this article are included in the article's Creative Commons licence, unless indicated otherwise in a credit line to the material. If material is not included in the article's Creative Commons licence and your intended use is not permitted by statutory regulation or exceeds the permitted use, you will need to obtain permission directly from the copyright holder. To view a copy of this licence, visit http://creativecommons.org/licenses/by/4.0/. 
shortest axial diameter reaches $11 \mathrm{~mm}$ in the jugulodigastric nodes and $10 \mathrm{~mm}$ in other cervical regions [6-8]. The sign "grouping" or clustering is also used. These signs are applied if there is a group of 3 or more nodes in a drainage region of a certain primary cancer [6-8]. The sign of clustering signifies an adverse predictive outcome and appears to be especially prevalent in head and neck cancer, mostly in squamous cell carcinoma SCC deposits [9-11]. Regarding the sign of shape, round nodes or those with lost fatty hila are also more likely to harbor metastases than oval bean-shaped nodes [12-15]. Although shape criteria by imaging were mainly designated for the context of enlarged nodes, nearly $82-86 \%$ of metastatic lymph nodes may be round but less than $10 \mathrm{~mm}$ Therefore, although roundness alone does not necessarily points out to metastasis [16].

A fourth imaging criterion is Inhomogeneity or central necrosis. Central necrosis is the imaging finding that is most reliably associated with metastasis and is reported to be present in nearly one-third of all metastatic cervical lymph nodes. In the setting of head and neck cancer, a necrotic node is considered pathologic regardless of size and can be seen in sub-centimetric nodes [11, 12, 15]. Another imaging criterion to be considered is the presence of nodal enhancement. The nodal hyper-enhancement implies an increase in nodal vascularity. These hyper-enhancing nodes can be undermining a variety of hyper-vascular neoplasms as lymphoma, papillary, and medullar thyroid carcinoma, and Kaposi sarcoma. Differentiation from other non-neoplastic hyper-vascular nodes needs constellation of other imaging criteria, which may be similar or not to those implied in SCC nodes. Besides intense enhancement, intra-nodal calcifications, either coarse or punctate (in 50-69\%), and cystic changes (20\%) may be noted. In larger tumors, a CT scan may show a thyroid mass invading adjacent structures (trachea, esophagus) [16-20].

To the best of our knowledge, our research is only the second in English literature trying to discuss the role of enhanced neck CT in differential diagnosis between SCC and non-SCC using comparative statistical analysis, comparing variables obtained from some of the abovementioned imaging criteria. SCC is the most common pathological type found in cervical lymph node metastasis followed by adenocarcinoma, the latter is the most common histological type in cervical lymph node metastasis from non-SCC. The purpose of our study was to assess the differential imaging findings of metastatic lymph nodes in SCC and nonSCC on neck CT and to facilitate the identification of primary focus before performing a biopsy and tailoring the most appropriate imaging plan when certain occult is suspected.

\section{Methods \\ Description of the retrospective study Ethics approval and consent to participate}

All procedures followed were in accordance with the ethical standards of the responsible committee on human experimentation "Institutional Review Board (IRB)" on the 14th of February 2017, and with the Helsinki Declaration of 1964 and later versions. The Committee's reference number is unavailable (not applicable). No consent was obtained from the patients since it was a retrospective study.

\section{Selection of patients, inclusion and exclusion criteria}

We retrospectively analyzed 157 patients (90 men, 67 women, and mean age 60 of age range between 17-90 years). The study was meant to include the adult ages of both sexes. Primary and recurrent post-operative cases, as well as post-irradiated head and neck cancers (more than 3 months from last radiation session) were included. Also, patients with known suggested primaries of head and neck or any other site of the body were included. The study excluded the following: patients with recent radiation history $<3$ months from last radiation session (to avoid masquerading changes of radiation), patients with lymphoma, prior recent FNC or any surgical interventions, and patients with signs of suggestive of inflammatory process. Also, patients with proven or suggested TB of the chest are excluded (refer to Table 1 on distribution of nodal groups with demographic data).

\section{Sites of primaries}

The number of SCC patients was 94 (59.9\%), and the number of non-SCC patients was 63 (40.1\%). The patients underwent a dedicated neck CT from April 2017 to April 2019. Of those 139 (88.5\%) had primary cancers originating from a head and neck sub-site, while 18 (11.5\%) had primary cancer outside the head and neck regions. SCC malignancies were distributed as follows: oral cavity $=13(13.8 \%)$, oropharynx $=4(4.3 \%)$, hypopharynx $=37(39.4 \%), \mathrm{NPC}=3(3.2 \%)$, larynx $=15$ $(16 \%)$, lung $=8(8.5 \%)$, skin $=8(8.5 \%)$, laryngectomy beds $=4(4.3 \%)$, others $=2(2.1 \%)$ including two SCCs of scalp. The non-SCC carcinomas include the following: oral cavity $=9(14.3 \%)$, including 7 melanomas and 2 adenocarcinomas, thyroid $=36(57.1 \%)$, oropharynx $=5$ (7.9\%), NPC $=2(3.2 \%)$, made of two adenocarcinomas, sino-nasal $=2(3.2 \%)$, lung small cell cancer $=1(1.6 \%)$, and skin melanoma $=1(1.6 \%), 7(11.1 \%)$. These others include one testicular, one ovarian, four breasts, and last one unknown (adenocarcinoma) (please refer to summary of distribution of primary sites as compared to SCC and non-SCC at Table 2).

Oral cavity patients had selective neck dissection while others underwent incisional biopsies of most suspicious 
Table 1 Distribution of pathologies among two groups of patients

\begin{tabular}{lll}
\hline & SCC & Non-SCC \\
\hline $\begin{array}{l}\text { Total number of cases } \\
\begin{array}{l}\text { Number of cases according to } \\
\text { underlying pathology }\end{array}\end{array}$ & $\begin{array}{l}\text { Hypopharynx 37, oral cavity/oropharynx 22, larynx 15, } \\
\text { lung 9, laryngectomies 3, skin SCC 8 }\end{array}$ & $\begin{array}{l}\text { Thyroid 36, nasopharyngeal carcinoma 10, sino-nasal 2, } \\
\text { cutaneous melanoma 1, others 15 }\end{array}$ \\
\hline
\end{tabular}

node (methods included direct FNC or US guided). However, needle biopsies were not correlated to open surgical results.

\section{Imaging examination technique}

All CT scans were performed with 128-multi-channel (Toshiba-Aquillion). Scanning parameters were as follows: 1 table pitch, $120 \mathrm{kVp}, 140 \mathrm{mAs}$, and $1.5 \mathrm{~mm}$ thickness. A $100 \mathrm{~mL}$ of nonionic contrast material (Omnipaque $350 \mathrm{mg} / \mathrm{ml}$ ) was injected at by a bi-phasic mode of injection followed by a $20 \mathrm{~mL}$ saline flush.

\section{Imaging analysis}

A head and neck radiologist with 12-year experience in head and neck radiology subspecialty reviewed two sets (SCC and non-SCC) of neck CT. The task was to assess the characteristics of the abnormal lymph nodes (number, margin types, enhancement patterns, size, bilaterality, necrosis, and grouping).

The following imaging criteria were evaluated:

1) Laterality: unilateral (ipsilateral or contra-lateral) versus "bilateral" versus "midline" disease is reported.
2) Node size: this is reported in axial plane measuring both short and long-axis diameter encompassing the outer margins.

3) "Levels" and "groups" of nodes involved: nodal grouping and levels according to known classification, adopted by "site Classification for Lymph Nodes From American Head and Neck Society and American Academy of OtolaryngologyHead and Neck Surgery" [21, 22].

4) Periphery/margin of nodes: these are reported as "irregular" versus "regular." No comment was made on extra-capsular margins as this hard to correlate with biopsy after operative excision.

5) Shape of the node: these include rounded, oblong, oval, and oval to oblong. The rounded node is defined as that node having a long-to-short axis ratio of less than $2 \mathrm{~mm}$. The oblong node is having a long-to-short axis ratio of more than $2 \mathrm{~mm}$ and more prominent in appearance than oval, while the oval is the normal expected pea-shaped node with or without loss of margin.

6) Grouping/clustering of drainage nodes: this image criterion is coined to multiple involved nodes; groups of $>3$ lymph nodes at any given nodal

Table 2 Comparison between the two studied groups according to demographic data

\begin{tabular}{|c|c|c|c|c|}
\hline & \multicolumn{2}{|l|}{ SCC } & \multirow[t]{2}{*}{ Test of sig. } & \multirow[t]{2}{*}{$p$} \\
\hline & $\operatorname{SCC}(n=94)$ & Non-SCC $(n=63)$ & & \\
\hline \multicolumn{5}{|l|}{ Groups } \\
\hline Neck & $84(89.4 \%)$ & $55(87.3 \%)$ & $x^{2}=0.158$ & 0.691 \\
\hline Non-neck & $10(10.6 \%)$ & $8(12.7 \%)$ & & \\
\hline \multicolumn{5}{|l|}{ Sex } \\
\hline Male & $72(76.6 \%)$ & $25(39.7 \%)$ & $x^{2}=21.768^{*}$ & $<0.001^{*}$ \\
\hline Female & $22(23.4 \%)$ & $38(60.3 \%)$ & & \\
\hline \multicolumn{5}{|l|}{ Age (years) } \\
\hline$<30$ & $1(1.1 \%)$ & $10(15.9 \%)$ & $x^{2}=28.979^{*}$ & $<0.001^{*}$ \\
\hline $30-39$ & $2(2.1 \%)$ & $9(14.3 \%)$ & & \\
\hline $40-49$ & $11(11.7 \%)$ & $13(20.6 \%)$ & & \\
\hline $50-59$ & $21(22.3 \%)$ & 10 (15.9\%) & & \\
\hline $60-69$ & $38(40.4 \%)$ & $13(20.6 \%)$ & & \\
\hline $70+$ & $21(22.3 \%)$ & $8(12.7 \%)$ & & \\
\hline Median (min.-max.) & $64(21-90)$ & $47(17-86)$ & $t=5.119^{*}$ & $<0.001^{*}$ \\
\hline Mean \pm SD. & $61.68 \pm 12.22$ & $48.57 \pm 17.7$ & & \\
\hline
\end{tabular}

$x^{2}$ chi square test, $t$ Student's $t$ test, $p p$ value for comparing between the two studied groups

*Statistically significant at $p \leq 0.05$ 
drainage level. The grouped/clustered nodes are further sub-classified into "conglomerate" versus "non-conglomerated" nodes. Conglomeration of lymph nodes was considered as juxtaposed lymph nodes; nearly sharing stuck-together walls that cannot be clearly delineated from each other with effacement of intervening fat.

7) Presence or absence of necrosis: necrosis is defined as the presence of intra-nodal identified $>2 \mathrm{~mm}$ internal hypo-density, showing a near water attenuation on CT (10-25 HU). Even few small foci in an essentially solid node will make this node termed a necrotic node. As regards the necrosis, the nodes are further sub-classified into (A) "Necrotic", (B) "non-Necrotic," and (C) "Cystic." These are further evaluated as follows:

A) The necrotic nodes are further analyzed as regards the following imaging criteria related to the necrosis, including the following (summarized in Fig. 1):

a) The epi-center of necrosis within nodal substance: central versus peripheral.

b) Thickness of margin of necrosis within the node is defined as thin $<1 \mathrm{~mm}$, thick $1-3 \mathrm{~mm}$; very thick > $3 \mathrm{~mm}$.

c) The inner margin of necrosis is defined as regular versus irregular.

d) Heterogeneity versus homogeneity: the necrosis itself is considered as heterogeneous if any of these criteria is seen: septa, debris, globulin, calcifications, or nodules.

e) The enhancement of this peripheral margin:

i. Iso-enhancement if near to the attenuation of head and neck muscles ii. Mild to moderate hyper-enhancement ( $>$ muscle and $<$ thyroid)

iii. Very hyper-enhancing: equal to or above thyroid or vessel enhancement

B) Non-necrotic lymph nodes were further subclassified according to "Homo/Heterogeneity" of enhancement, as those showing homogeneous or heterogeneous enhancement. "Degree of enhancement" of the node is sub-classified as follows: (1) iso-enhancement if near to the attenuation of head and neck muscles, (2) moderate hyper-enhancement is higher than muscle and less than thyroid, (3) very hyperenhancing: equal to or above thyroid and vessel enhancement.

C) Cystic nodes: The term cystic is confined only to near wholly necrotic nodes with near homogenous pure fluid attenuation and thin < $0.1 \mathrm{~mm}$ nodal margin or even imperceptible. These are analyzed further into presence or absence of calcification or solid nodules.

\section{Statistical analysis of the data}

Statistical analysis of the data with comparison of imaging variable between SCC and non-SCC nodal groups: data were fed to the computer and analyzed using the IBM SPSS software package version 20.0 (Armonk, NY: IBM Corp). Qualitative data were described using number and percent. Kolmogorov-Smirnov test was used to verify the normality of distribution. Quantitative data were described using range (minimum and maximum), mean, standard deviation, and median. The significance of the obtained results was judged at $5 \%$ level. The used tests were (1) chi-square test for categorical variables, to compare between different groups, (2) Fisher's exact or Monte Carlo correction, (3) Student's $t$ test.

\section{Results}

Regarding the laterality of nodes, the majority of 131 (83.4\%) had unilateral disease, only two of them had
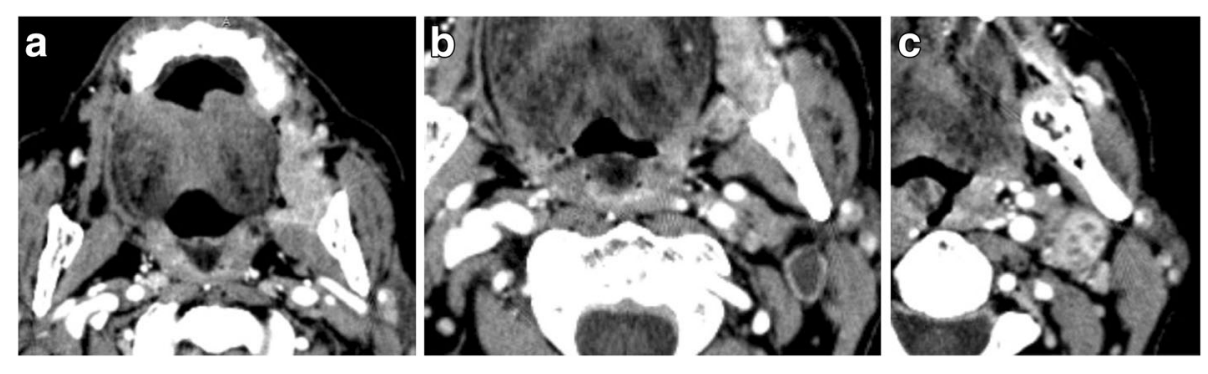

Fig. 1 A case of retro-molar molar trigone squamous cell carcinoma (yellow arrow in " a"), with associated metastatic necrotic nodes at level II (b), showing thin peripheral margin and is not termed cystic in our study. This shows thin rim with regular margin. Other nodes are more solid with tiny necrotic foci and moderate hyperenhancement (c). The features are all in favor of squamous cell carcinoma node 
isolated contra-lateral disease in two patients with oral cavity primary. Bilateral disease was seen in 23 (14.6\%). Isolated midline disease was seen in only $3(1.9 \%)$, two of them had single Delphian nodes in thyroid cancers, and a single midline sub-mental node was also observed in oral cancer case.

As expected, the jugulodigastric-JGD (level II) nodes had the greatest burden disease being involved in the highest ratio of patients (92 patients IIA and 63 for IIB, representing $=58.6 \%$ and $40.1 \%$ respectively). These nodes represent the sentinel nodes of head and neck cancer. All involved nodes came from primary head and neck cancers apart from single cases of breast cancer with extensive supra-clavicular and deep cervical nodes. Level I nodes had the second-highest prevalence, counted as $57(36.3 \%)$ for IA and 25 (15.9\%) for level IB. Their primaries are prevalent in the oral cavity (oral tongue, gingiva, and alveolar margin). Level III nodes had the third-highest prevalence, 57 (36.3\%), usually noted in association with level II nodes, and not in isolation.

Level IVA was seen in 15 cases (9.6\%) and level IVB in 30 cases (19.1\%). Those were never seen isolated. They were involved in thyroid cancer in combination with levels III and supra clavicular or with level II (JDG) in the oral cavity and oropharynx after crossing the sentinel II nodes. Level V was involved in 12 (7.6\%), 4 were in the five cases of NPC, a single case atypically involved in oropharyngeal mass with extensive deep cervical adenopathies, 4 were thyroid cancers, and the remainder are primaries outside the head and neck regions.

Level VI nodes were involved in 12 patients (7.6\%), seen exclusively in visceral space malignancies (i.e., thyroid). Parotid nodes were involved in 5 (3.2\%), all of which originated from superficial tumors (skin). Isolated para-pharyngeal space adenopathies were seen in 4 (2.5\%), all had primary thyroid cancers. Retropharyngeal adenopathies were never seen in isolations. These were involved in 12 (7.6\%); they were seen in oropharyngeal, NPC, sino-nasal, and thyroid cancers. The supra clavicular had high prevalence, seen in 26 patients (16.6\%): these involved all the 18 patients with primaries outside the heads and neck regions. However, the thyroid gland had the highest prevalence of supra-clavicular metastatic nodes and was always involved with level III or IV nodes in the same patient. Delphian node was involved in 8 patients; all had primary thyroid cancer.

Comparing nodal level and group involvement between SCC and non-SCC revealed: Involvement of JGD, sub-mental, and supra-clavicular nodes had statistically significant difference among SCC and non-SCC groups. This is well expected as JGD represents the nodal sentinel drainage of upper aero-digestive SCC lesions mostly represented by the oral cavity and oropharynx. The involvement of level II was shown in 67 (71.3\%) of SCC groups compared to 25 (39.7\%) in non-SCC, while level IIB was seen in 41 (43.6\%) compared to 22 (24\%). A key difference between both groups is that involvement of level IIA/B in SCC could occur solely or in combination with level I or less likely with more caudal nodal levels. On the other hand, involvement of level IIA/B in nonSCC always occurred in combination with other more caudal "sentinel" levels including III, IVA/B, and supraclavicular nodes. Involvement of supra-clavicular nodal groups also had statistical significance between both groups, being higher in non-SCC groups $(15(23.8 \%))$ as compared to $11(11.7 \%)$ in SCC groups (Table 3 shows laterality, nodal levels, and groups involved).

The study showed multiple-nodal involvement in 94 (59.9\%) of total 157 cases, as compared to 63 (40.1\%) of single-nodal involvement. Out of the "multiple nodal Involvement," the sign of "grouping" was seen in 69 cases: 56 (59.6\%) showed grouping without conglomeration, while 13 (13.8\%) showing grouping and conglomeration. Six out of conglomerated nodes (total are 13) had primary thyroid cancers while others had primaries of oral cavity, oropharynx, and hypo-pharynx as well as single case of testicular cancer (non-SCC). The non-grouping was seen in 25 (26.6\%). The "grouping" of nodes usually points to metastatic nature. However, this sign of grouping plus or minus conglomeration had no statistical significance among the SCC and non-SCC groups (Table 4). Figures 2, 3, 4, 5, and 6 show illustrated cases of SCC and non-SCC metastatic nodal groups and how constellation of described imaging criteria is used in combination to differentiate the two categories on $\mathrm{CT}$ basis.

Necrotic nodes as defined in our study showed higher prevalence among metastatic nodes in both groups constituting a total of $107(68.2 \%)$ of the total case toll, as compared to "non-necrotic" solid nodes 31 (19.7\%), and "cystic" nodes 19 (12.1\%). The necrosis was almost central in the highest majority of cases (95.3\%). Margins of necrosis with different thickness and regularities were evaluated: combination of thick and irregular margin had the highest incidence of 49 (45.8\%) among the total number of cases, followed by thick regular margin (29.9\%). Heterogeneity of necrosis itself had prevalence over homogenous ones (72\%). Statistical comparison between SCC and non-SCC groups showed that the criterion of necrosis itself was statistically significant in SCC as compared to non-SCC $(n=74: 78.7 \%)$ in SCCs compared to $(n=33: 52.4 \%)$ in non-SCC. The rim of enhancement around the necrosis was mostly isoenhancing in SCC nodes, as compared to non-SCC (hyper to very hyper). This sign also had some statistically significant difference (Table 5). 
Table 3 Comparison between the two studied groups according to distribution (laterality and nodal level)

\begin{tabular}{|c|c|c|c|c|}
\hline & \multicolumn{2}{|l|}{ SCC } & \multirow[t]{2}{*}{$x^{2}$} & \multirow[t]{2}{*}{$p$} \\
\hline & SCC $(n=94)$ & Non-SCC $(n=63)$ & & \\
\hline \multicolumn{5}{|l|}{ Laterality } \\
\hline Unilateral & 77 (81.9\%) & $54(85.7 \%)$ & 1.876 & ${ }^{M C} C_{p}=0.380$ \\
\hline Midline & $1(1.1 \%)$ & $2(3.2 \%)$ & & \\
\hline Bilateral & $16(17 \%)$ & $7(11.1 \%)$ & & \\
\hline \multicolumn{5}{|c|}{ Involved nodal groups and levels } \\
\hline $\mathrm{IA}$ & $27(28.7 \%)$ & $30(47.6 \%)$ & $5.824^{*}$ & $0.016^{*}$ \\
\hline $\mathrm{IB}$ & $21(22.3 \%)$ & $4(6.3 \%)$ & $7.205^{*}$ & $0.007^{*}$ \\
\hline$\| \mathrm{A}$ & $67(71.3 \%)$ & $25(39.7 \%)$ & $15.519^{*}$ & $<0.001^{*}$ \\
\hline$\| \mathrm{B}$ & $41(43.6 \%)$ & $22(34.9 \%)$ & 1.187 & 0.276 \\
\hline III & $25(26.6 \%)$ & $32(50.8 \%)$ & $9.551^{*}$ & $0.002^{*}$ \\
\hline IVA & $3(3.2 \%)$ & $12(19 \%)$ & $10.974^{*}$ & $0.001^{*}$ \\
\hline IVB & $3(3.2 \%)$ & $27(42.9 \%)$ & $38.395^{*}$ & $<0.001^{*}$ \\
\hline V & $3(3.2 \%)$ & $9(14.3 \%)$ & $6.577^{*}$ & $\mathrm{FE}_{p}=0.014^{*}$ \\
\hline $\mathrm{Vl}$ & $5(5.3 \%)$ & $7(11.1 \%)$ & 1.793 & ${ }^{\mathrm{FE}_{p}}=0.225$ \\
\hline Parotid & $5(5.3 \%)$ & $0(0 \%)$ & 3.461 & $\mathrm{FE}_{p}=0.083$ \\
\hline Para pharyngeal & $1(1.1 \%)$ & $3(4.8 \%)$ & 2.078 & $\mathrm{FE}_{p}=0.303$ \\
\hline Pre auricular & $1(1.1 \%)$ & $0(0 \%)$ & 0.675 & $\mathrm{FE}_{p}=1.000$ \\
\hline Retropharyngeal & $7(7.4 \%)$ & $5(7.9 \%)$ & 0.013 & $\mathrm{FE}_{p}=1.000$ \\
\hline Peri vertebral & $1(1.1 \%)$ & $1(1.6 \%)$ & 0.082 & $\mathrm{FE}_{p}=1.000$ \\
\hline Supra clavicualr & $11(11.7 \%)$ & $15(23.8 \%)$ & $4.002^{*}$ & $0.045^{*}$ \\
\hline Dissection bed & $1(1.1 \%)$ & $1(1.6 \%)$ & 0.082 & $\mathrm{FE}_{p}=1.000$ \\
\hline Mediastinal & $0(0 \%)$ & $2(3.2 \%)$ & 3.023 & $\mathrm{FE}_{p}=0.159$ \\
\hline Delphian & $0(0 \%)$ & $8(12.7 \%)$ & $12.577^{*}$ & ${ }^{\mathrm{FE}} p=0.001^{*}$ \\
\hline EJV & $9(9.6 \%)$ & $0(0 \%)$ & $6.399^{*}$ & ${ }^{\mathrm{FE}} p=0.011^{*}$ \\
\hline
\end{tabular}

$x^{2}$ chi-square test, MC Monte Carlo, FE Fisher's exact, $p p$ value for comparing between the two studied groups

*Statistically significant at $p \leq 0.05$

Cystic nodes had a very good statistical significance among both groups: They were found in 3 (3.2\%) of SCC patients, and in 16 (25.4\%) of non-SCC patients. The SCC cystic nodes had prevalence in oral cavity nodes while non-SCC cystic nodes were mainly from thyroid primaries, apart from single testicular cases. However, intrinsic imaging details of a cystic node can adequately separate thyroid form non-thyroid metastatic adenopathies. Thyroid papillary nodes show with cystic nodes showed combination with one of two: calcification and/or moderate or very hyper-enhancement in mural nodules or peripheral perceptible rims. The medullary and anaplastic metastatic nodes in our study showed mainly non-necrotic solid adenopathies with variable degrees of mild to very hyper-enchantment.

Non-necrotic nodes had no statistical significance between the two groups, counted as $17(18.1 \%)$ in SCC nodes compared to $14(22.2 \%)$ in non-SCC nodes. Still, intrinsic imaging criteria of solid non-necrotic nodes have not been detailed in our study. Still, solid metastases of thyroid primaries had variable degrees of hyper-enhancement (mild to very hyper-enhancement). The periphery of nodes was mostly regular (73 (77.7\%)) of SCC and $48(76.2 \%)$ in non-SCC. This shows that marginal irregularity is not highly prevalent in metastatic nodes altogether, in contra-distinction to non-neoplastic inflammatory nodes where peri-nodal inflammation and peri-adenitis is usually prevailing (Table 6).

\section{Discussion}

To the best of our knowledge, only a single previous published research has discussed the role of enhanced neck CT in differentiation between SCC and non-SCC metastatic nodes. This was made by Lee NY et al. [23] who performed a similar retrospective study on a slightly smaller sample of patients including a total of $105 \mathrm{pa}-$ tients, encompassing both SCC and non-SCC groups. In their study, the SCC patients constituted a smaller percentage of 46 patients compared to 50 SCC patients. In contrary, our study had much higher SCC patients 94 
Table 4 Comparison between the two studied groups according to different imaging parameters

\begin{tabular}{|c|c|c|c|c|}
\hline & \multicolumn{2}{|l|}{ SCC } & \multirow[t]{2}{*}{$x^{2}$} & \multirow[t]{2}{*}{$p$} \\
\hline & $\operatorname{SCC}(n=94)$ & Non-SCC $(n=63)$ & & \\
\hline \multicolumn{5}{|l|}{ Necrotic } \\
\hline Non-necrotic & $17(18.1 \%)$ & $14(22.2 \%)$ & $19.536^{*}$ & $<0.001^{*}$ \\
\hline Necrotic & $74(78.7 \%)$ & $33(52.4 \%)$ & & \\
\hline Cystic & $3(3.2 \%)$ & $16(25.4 \%)$ & & \\
\hline Necrosis central/peripheral $(n=107)$ & $(n=74)$ & $(n=33)$ & & \\
\hline Central & $72(97.3 \%)$ & 30 (90.9\%) & 4.030 & ${ }^{\mathrm{MC}} p=0.116$ \\
\hline Peripheral & $0(0 \%)$ & $2(6.1 \%)$ & & \\
\hline Central and peripheral & $2(2.7 \%)$ & $1(3 \%)$ & & \\
\hline Margin regularity $(n=107)$ & $(n=74)$ & $(n=33)$ & & \\
\hline Irregular & $41(55.4 \%)$ & $16(48.5 \%)$ & 0.439 & 0.508 \\
\hline Regular & $33(44.6 \%)$ & $17(51.5 \%)$ & & \\
\hline Enhancing margin thickness $(n=107)$ & $(n=74)$ & $(n=33)$ & & \\
\hline Thin & $13(17.6 \%)$ & $5(15.2 \%)$ & 0.246 & 0.884 \\
\hline Thick & $56(75.7 \%)$ & $25(75.8 \%)$ & & \\
\hline Very thick & $5(6.8 \%)$ & $3(9.1 \%)$ & & \\
\hline Combined $(n=107)$ & $(n=74)$ & $(n=33)$ & & \\
\hline Irregular + thin & $3(4.1 \%)$ & $1(3 \%)$ & 2.292 & ${ }^{M C} C_{p}=0.867$ \\
\hline Irregular + thick & $36(48.6 \%)$ & $13(39.4 \%)$ & & \\
\hline Irregular + very thick & $2(2.7 \%)$ & $2(6.1 \%)$ & & \\
\hline Regular + thin & $10(13.5 \%)$ & $4(12.1 \%)$ & & \\
\hline Regular + thick & $20(27 \%)$ & $12(36.4 \%)$ & & \\
\hline Regular + very thick & $3(4.1 \%)$ & $1(3 \%)$ & & \\
\hline Pattern/heterogeneity of central necrosis $(n=107)$ & $(n=74)$ & $(n=33)$ & & \\
\hline Homogenous & $21(28.4 \%)$ & $9(27.3 \%)$ & 0.014 & 0.906 \\
\hline Heterogeneous & $53(71.6 \%)$ & $24(72.7 \%)$ & & \\
\hline Degree of rim enhancement in necrotic node $(n=107)$ & $(n=74)$ & $(n=33)$ & & \\
\hline ISO & 68 (91.9\%) & $18(54.5 \%)$ & $24.112^{*}$ & ${ }^{\mathrm{MC}} p<0.001^{*}$ \\
\hline Hyper & $2(2.7 \%)$ & 8 (24.2\%) & & \\
\hline Mild hyper & $4(5.4 \%)$ & $2(6.1 \%)$ & & \\
\hline Very hyper & $0(0 \%)$ & 5 (15.2\%) & & \\
\hline
\end{tabular}

$x^{2}$ chi-square test, MC Monte Carlo, $p p$ value for comparing between the two studied groups

*Statistically significant at $p \leq 0.05$

(59.9\%), as compared to non-SCC 63 (40.1\%). This was nicely expected in view of the common incidence of head and neck SCC, being the most common histopathological subtypes. They included sites outside the head and neck (breast, cervix, gall bladder, prostate and colon, and single case of unknown primary). Our patients with distant sites included primaries of lung, breast, ovaries, testes, esophagus, and a neuroectodermal tumor as well as a single unknown primary adenocarcinoma. These constituted 18 (11.5\%) of the total study cases [23].

In their study, patients in the SCC group (36-89 years, mean: 63.0 years) were significantly older than those in the non-SCC group (16-81 years, mean: 56.1 years). This was in good correlation with our study: the SCC patients were older (64 (21-90 years), compared to non-SCC group 47 (17-86 years). In their study, Lee et al. [23] stated that various primary cancers were included in this study with a high proportion of young thyroid cancer cases in the non-SCC group $(22 / 50)$ correlated to this result. However, in our study, this was not the case since our thyroid cases include old-onset papillary carcinomas and anaplastic variant. Still, the oldest of patients of SCC occupied primaries of lung, oropharynx, hypopharynx, and larynx (> 60 years of age) [23]. 


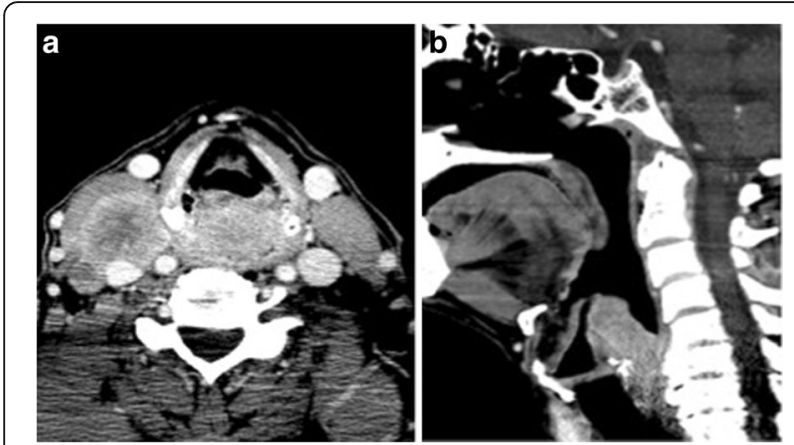

Fig. 2 A case of 82-year-old male patient with hypopharyngeal squamous cell carcinoma. This is associated with right-sided necrotic nodes at IIB and III. They show central necrosis at the largest node with a very thick irregular margin showing slight "hyper-enhancement," and grouping non-conglomerated appearance. (a) Shows the main tumor and nodal disease at two levels, while (b) image shows the longitudinal tumor. The features of "necrosis" are consistent with squamous cell carcinoma metastatic node according to our study

In their study, Lee et al. [23] stated that SCC was more frequent in men than in women (female to male ratio, 11:35) and non-SCC was relatively more frequent in women than in men (female to male ratio, 29:21). Our study also showed similar results, SCCs had prevalence in males $(76.6 \%)$, while the non-SCC had prevalence in females $(60.3 \%)$. Thyroid cancer was noted in 22 patients out of 50 non-SCCs in their study, as compared to 36 out of 36 non-SCCs in our study. Thyroid cancer was

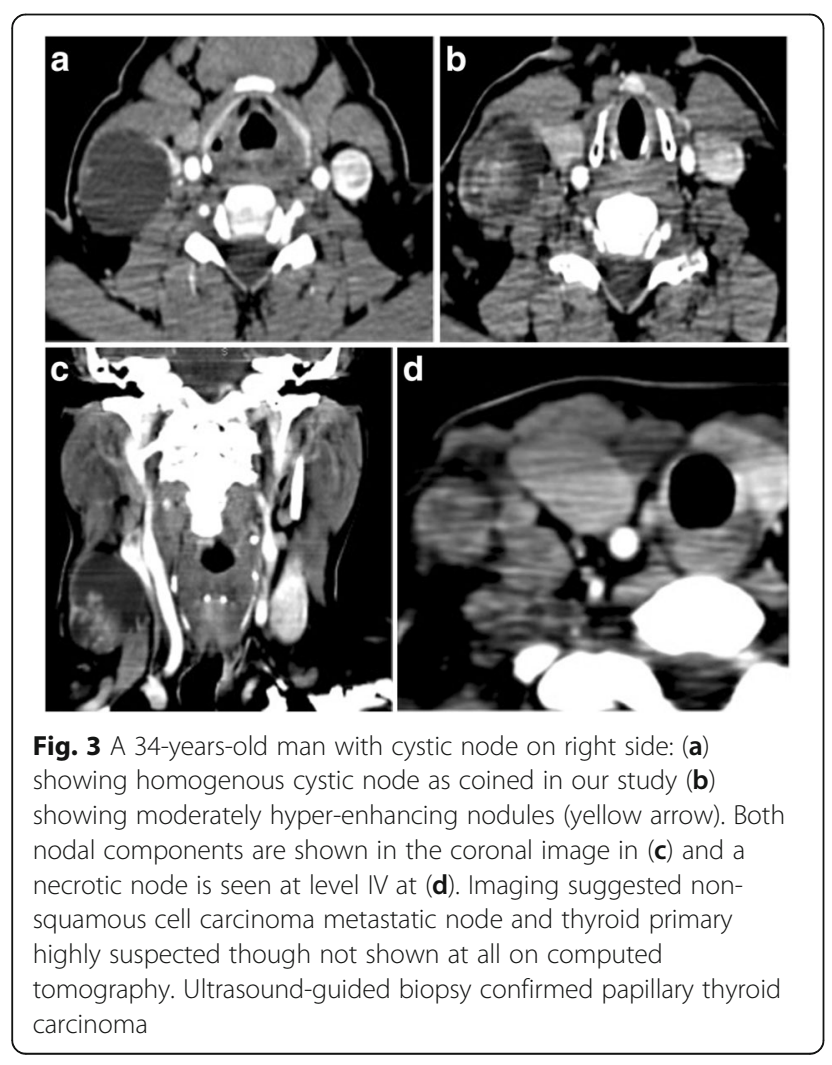

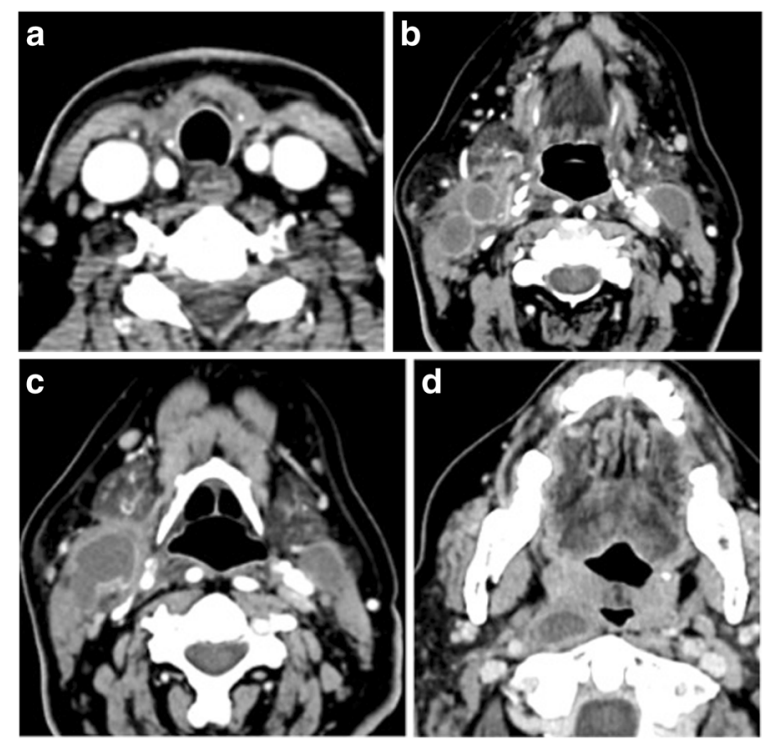

Fig. 4 A case of 63-year-woman of prior old (10 years ago) of operated thyroid cancer with multiple questionable cervical nodal swellings. (a) Shows clear thyroidectomy bed. (a and $\mathbf{b}$ ) Shows grouped bilateral markedly necrotic nodes with smooth regular iso to mild hyperenhancing smooth and thin margin, at levels II mainly with grouping and near conglomeration. A right-sided retropharyngeal node is seen (c). Features are in favor of being squamous cell carcinoma metastatic nodes over the non-squamous cell cancer nodes, putting down the possibility of thyroid recurrence. (d) Shows the primary oropharyngeal primary squamous cell carcinoma proved later. The signs helped to differentiate a new squamous cell carcinoma metastatic node and not a non-squamous cell "thyroid" metastatic node regarding the history of operated thyroid cancer

much more frequent in females than in males (female to male ratio, 18:4). In our study, thyroid cancer was also much more frequent in females than in males (female to male ratio, 19:7). In the previous reports by Muir et al. [24] and Voutilainen et al. [25] stated that the female-tomale ratio of thyroid cancer ranged from 2:1 to 5:1 in most populations [23-25].

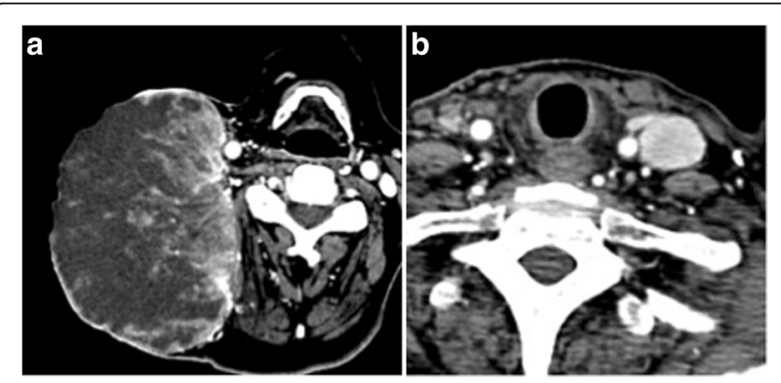

Fig. 5 A case of 67 years old of nodal recurrence from previous dedifferentiated thyroid carcinoma. A very huge invasive node on right side with marked necrosis (a); essentially not central and very thick hyper-enhancing irregular margin and small non-necrotic IV node on left side (b), showing moderate to very hyper-enhancement 


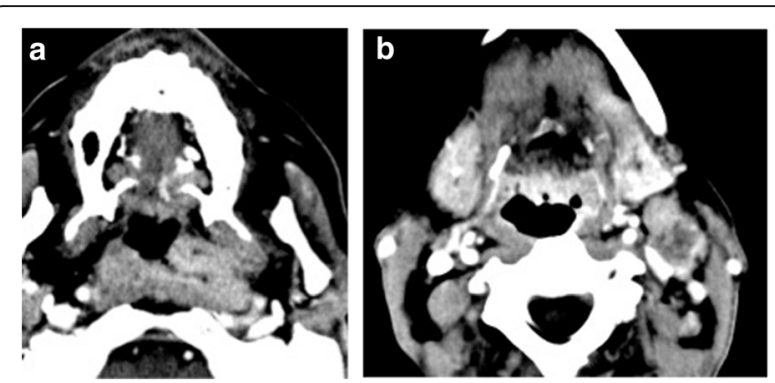

Fig. 6 A 25 years old female with asymmetric thickening of nasopharyngeal recess in a proven NPC (a) and necrotic SSC node at nodal level II on the left side (b)

In the study by Lee et al. [23], the number of lymph nodes in the non-SCC group and the number of lymph nodes smaller than $3 \mathrm{~cm}$ were greater than those in the SCC group. They reported a statistically significant difference between the SCC group and the non-SCC group except for cases of thyroid cancer. In our study, the measurement of nodal size was made only as part of routine work and not meant for being part of statistical analysis. However, a total of 39 patients had nodes greater than $3 \mathrm{~cm}$ in maximum axial diameter, 20 of them were SCC patients. The four cases in our study having $>6 \mathrm{~cm}$ longest axial diameter was seen in 3 nonSCC compared to single SCC. Evaluation of nodal size as part of characterization of nodal metastases or differentiation of SCC from non-SCC needs larger patient samples with trial to separate thyroid patients [23].

The nodal levels of IA and IB had statistical significance between the two groups. These are nearly solely involved in oral cavity and oropharyngeal cancers with or without upper deep cervical nodes (jugulodigastricJDG nodes). These would not show isolated involvement in cases of thyroid cancers without more caudal deep cervical nodes. Also, levels IVB and supra-clavicular nodes showed statistical difference, being involved in most of thyroid cancers with variable involvement of level VI, Delphian, or III. Isolated Delphian node involvement is nearly pathognomonic in thyroid cancers. Unexpectedly, the involvement of level VI had no statistical significance between the SCC and non-SCC. This could be attributed to the fact that the study included primary and post-operative cases. The operated cases as laryngectomies (SCC) may have non-regional nodal deposits at level VI due to altered and inter-mixed lymphatics following surgical dissections. However, the exclusive involvement of VI in the primary presentation of cervical nodal metastasis points to visceral space, non-SCC malignancy mostly of thyroid cancer.

The study of the criterion of grouping as a sign of malignancy in nodes revealed no statistical significance between the two groups. Grouping with conglomeration was seen in $30(58.8 \%)$ of SCC patients, compared to 26 (60.5\%) of non-SC groups. While grouping without conglomeration was seen in $6(11.8 \%)$, compared to 7 $(16.3 \%)$ of the non-SCC. This was attributed to the fact that conglomeration was seen in oral cavity SCC metastatic nodes and some types of thyroid cancer nodal metastases. This was in disagreement with results by Lee et al.; they coined the term of conglomeration to be equivalent to the description of simple grouping in our stud. They show statistical significance, being more commonly and more frequently observed in the SCC group $(55.6 \%)$ than in the non-SCC group (44.4\%). Conglomeration and grouping are used synonymously in literature; it is described as grouping of at least three lymph nodes and has been used as a morphologic criterion for the diagnosis of SCC of the head and neck region [26].

Previous reports have demonstrated that the detection of intra-nodal necrosis in patients with primary head and neck tumors is the most reliable sign of being a metastatic node [24, 27]. Necrosis is frequently seen in nodal metastases from SCC of the head and neck, and it was used for differentiation form in non-metastatic nodes $[8,28,29]$. In the study by Lee NY et al. [23], they

Table 5 Comparison between the two studied groups according to different parameters: margins and shape

\begin{tabular}{|c|c|c|c|c|}
\hline & \multicolumn{2}{|l|}{ SCC } & \multirow[t]{2}{*}{$x^{2}$} & \multirow[t]{2}{*}{$p$} \\
\hline & SCC $(n=94)$ & Non-SCC $(n=63)$ & & \\
\hline \multicolumn{5}{|c|}{ Outer margins/periphery of node } \\
\hline Regular & $73(77.7 \%)$ & $48(76.2 \%)$ & 0.046 & 0.830 \\
\hline Irregular & $21(22.3 \%)$ & $15(23.8 \%)$ & & \\
\hline \multicolumn{5}{|l|}{ Shape } \\
\hline Rounded & $80(85.1 \%)$ & $53(84.1 \%)$ & $10.307^{*}$ & ${ }^{M C} p=0.008^{*}$ \\
\hline Oblong & $4(4.3 \%)$ & $9(14.3 \%)$ & & \\
\hline Oval & $8(8.5 \%)$ & $0(0 \%)$ & & \\
\hline Oval to oblong & $2(2.1 \%)$ & $1(1.6 \%)$ & & \\
\hline
\end{tabular}

$x^{2}$ chi-square test, MC Monte Carlo, $p p$ value for comparing between the two studied groups

*Statistically significant at $p \leq 0.05$ 
Table 6 Comparison between the two studied groups according to grouping

\begin{tabular}{|c|c|c|c|c|}
\hline \multirow[t]{2}{*}{ Grouping } & \multicolumn{2}{|l|}{ SCC } & \multirow[t]{2}{*}{$x^{2}$} & \multirow[t]{2}{*}{$p$} \\
\hline & SCC $(n=94)$ & Non-SCC $(n=63)$ & & \\
\hline Single & $43(45.7 \%)$ & $20(31.7 \%)$ & 3.077 & 0.079 \\
\hline Multiple & $51(54.3 \%)$ & $43(68.3 \%)$ & & \\
\hline If Multiple $(n=94)$ & $(n=51)$ & $(n=43)$ & & \\
\hline No grouping & $15(29.4 \%)$ & $10(23.3 \%)$ & 0.687 & 0.709 \\
\hline Grouping & $30(58.8 \%)$ & $26(60.5 \%)$ & & \\
\hline Grouping-conglomeration & $6(11.8 \%)$ & $7(16.3 \%)$ & & \\
\hline
\end{tabular}

$x^{2}$ chi-square test, $p p$ value for comparing between the two studied groups

revealed no significant difference in the presence of necrosis between the two groups. In disagreement with their study, ours revealed good statistical significance of the sign of necrosis between the two groups, showing high prevalence between the two groups, occurring in 74 (78.7\%) of the SCC patients compared to 33 (52.4\%) of non-SCC. A good explanation for this disagreement is that their study did not separate the cystic form necrotic nodes. In our study, cystic nodes are considered as a separate group and are further sub-classified according to combination with fine calcifications and hyperenhancing wall or nodules. Please refer to Figs. 2, 3, 4, 5, and 6 for comparison of nodal groups.

When analyzing the intrinsic details of nodal morphology regarding nodal necrosis, our study tried to analyze different combinations of marginal thickness and smoothness versus regularities. The combination of thick margin with either regularity or irregularities had the highest prevalence between the two groups. Similarly, Lee et al. [23] pointed out that there was no significant difference in the ratio of lymph nodes with thick irregular wall enhancement to total lymph nodes between the SCC group and the non-SC group except for cases of thyroid cancer.

Our study had several limitations. First, the total sample size, though larger than our preceding example made by Lee et al. [23], still larger group of patients could give more significant statistical results. Second, this is a retrospective review of patients who underwent imaging investigations as part of the assessment for head and neck metastasis. Third, pathological confirmation by operative biopsy was not achieved in all patients. Fourth, thyroid cancer metastatic nodes are supposed to have distinct features among the non-SCC and these are recommended for further evaluation in separate consideration and in larger group of patients.

\section{Conclusion}

In conclusion, a combination of metastatic-looking adenopathies in certain nodal groups could suggest underlying squamous cell carcinoma or non-squamous cell carcinoma. Nodal necrosis, separating the "cystic" group, and involvement of nodal levels II and I in isolation from caudal deep cervical nodes in older aged men; both were statistically significant in the SCC compared to non-SCC. Though not showing statistical difference, the grouping $+/$ - conglomeration and multiple or non-single nodal group/level involvement could show statistical significance with larger study size and separation of thyroid metastatic group.

\section{Abbreviations}

CT: Computed tomography; SCC: Squamous cell carcinoma; Non-SCC: Nonsquamous cell carcinoma; US: Ultrasound; MRI: Magnetic resonance imaging; HU: Hounsfield unit; NPC: Naso-pharyngeal carcinoma

\section{Acknowledgements}

Not applicable.

\section{Authors' contributions}

AM provided the cases and final diagnoses, with detailed description of results. LE gave the idea, wrote the section of introduction, and provided the whole references for introduction and discussion with making of figure legends. "All authors read and approved the final manuscript."

\section{Funding}

This study had no funding from any resource (none).

\section{Availability of data and materials}

The datasets used and/or analyzed during the current study are available from the corresponding author on reasonable request.

\section{Ethics approval and consent to participate}

All procedures followed were in accordance with the ethical standards of the responsible committee on human experimentation "Institutional Review Board (IRB)" of Alexandria General Hospital on the 14th of February 2017, and with the Helsinki Declaration of 1964 and later versions. The committee's reference number is unavailable (not applicable). No consent was obtained from the patients since it was a retrospective study.

\section{Consent for publication}

All patients included in this research gave written informed consent to publish the data contained within this study.

\section{Competing interests}

The authors declare that they have no competing interests.

\section{Author details}

${ }^{1}$ Department of Diagnostic and Interventional Radiology, Alexandria Faculty of Medicine, Alexandria 21131, Egypt. ${ }^{2}$ Department of Oto-rhino-laryngology, Alexandria Faculty of Medicine, Alexandria, Egypt. 
Received: 27 March 2020 Accepted: 29 September 2020

Published online: 30 October 2020

\section{References}

1. Seethala RR (2009) Current state of neck dissection in the United States. Head Neck Pathology 3:238-245

2. Dunne A, Muller HH, Eisele DW, Kebel K, Moll R, Werner JA (2006) Meta-analysis of the prognostic significance of per nodal spread in head and neck squamous cell carcinomas (HNSCC) patients. Eur J Cancer 42(12):1863-1868

3. Snow GB, Patel P, Leemans CR, Tiwari R (1992) Management of cervical lymph nodes in patients with head and neck cancer. Eur Arch Otorhinolaryngol 249:187-194

4. Brown AE, Langdon JD (1995) Management of oral cancer. Ann R Coll Surg Engl 77:404-408

5. Miller JE, Al-Attar NC, Brown OH, Shaughness GG, Rosculet NP, Avram AM, Hughes DT (2018) Location and causation of residual lymph node metastases after surgical treatment of regionally advanced differentiated thyroid cancer. Thyroid 28:593-600

6. Johnson J (1990) A surgeon looks at cervical lymph nodes. Radiology 175: 607-610

7. $\mathrm{Ng} \mathrm{SH}, \mathrm{Ko} \mathrm{SH}$, Toh $\mathrm{CH}$, Chen YL (2006) Imaging of neck metastases. Med J 29(2):119-126

8. Brekel MW, Stel HV, Castelijns JA, Nauta JJ, van der Waal I, Valk J, Meyer CJ, Snow GB (1990) Cervical lymph node metastasis: assessment of radiologic criteria. Radiology 177:379-384

9. Imhof H, Czerny C, Dirisamer A (2003) Head and neck imaging with MDCT. Eur J Radiol 45:23-31

10. Curtin HD, Ishwaran H, Mancuso AA, Dalley RW, Caudry DJ, McNeil BJ (1998) Comparison of $C T$ and MR imaging in staging of neck metastases. Radiology 207:123-130

11. Som PM (1992) Detection of metastasis in cervical lymph nodes: CT and MR criteria and differential diagnosis. AJR Am J Roentgenol 158:961-969

12. Lee KJ, Kirsch C, Sayre J (2008) Lymph node clustering in head and neck squamous cell cancer. Otolaryngol Head Neck Surgery 139:38-40

13. Stoeckli SJ, Pfaltz M, Steinert H (2002) Histopathological features of occult metastasis detected by sentinel lymph node biopsy in oral and oropharyngeal squamous cell carcinoma. Laryngoscope 112:111-115

14. Xu C, Liu Y, Wang P (2010) Integrative analysis of DNA copy number and gene expression in metastatic oral squamous cell carcinoma identifies genes associated with poor survival. Mol Cancer 9:143

15. Kuna SK, Bracic I, Tesic V (2006) Ultrasonographic differentiation of benign from malignant neck lymphadenopathy in thyroid cancer. J Ultrasound Med 25:1531-1537

16. Dmytriw AA, El Beltagi A, Bartlett E, Sahgal A, Poon CS, MD FR, Fatterpekar G, Yu E (2014) CRISPS: a pictorial essay of an acronym to interpreting metastatic head and neck lymphadenopathy. Can Assoc Radiol J 65:232-241

17. Som PM, Brandwein M, Lidov M (1994) The varied presentations of papillary thyroid-carcinoma cervical nodal disease: CT and MR findings. Am J Radiol Neuroradiol 15:1123-1128

18. Cheng F, Yanyan C, Zhu L, Zhou B, Younghong Y, Chen Y, Wen C, Chen S (2019) Risk factors for cervical lymph node metastasis of papillary thyroid microcarcinoma: a single-center retrospective study. Int J Endocrinol 8:1-6

19. Karandikar A, Gummalla KM, Loke SC, Goh J, Tan TY (2016) Approach to intensely enhancing neck nodes. Diagn Interv Radiol 22:168-172

20. King AD (2008) Imaging for staging and management of thyroid cancer. Cancer Imaging 8:57-69

21. Hoang J, Benjamin JV, Ludwig J, Glastonbury CM (2013) Evaluation of cervical lymph nodes in head and neck cancer with CT and MRI: tips, traps, and a systematic approach. Am J Radiology 200:17-25

22. Som P, Curtin H, Mancuso A (2000) Imaging-based nodal classification for evaluation of neck metastatic adenopathy. Am J Radiol 174:837-844

23. Lee NY, Han JK, Kim HW, Shin HC, Kim YK, Jou SS (2015) Comparative study of lymph node metastasis from squamous cell carcinoma and nonsquamous cell carcinoma on neck CT. J Korean Soc Radiol 72(4):271-281

24. Parkin DM, Muir CS (1992) Cancer incidence in five continents. Comparability and quality of data. IARC Sci Publ 120:45-173

25. Voutilainen PE, Multanen MM, Leppäniemi AK, Haglund CH, Haapiainen RK, Franssila KO (2001) Prognosis after lymph node recurrence in papillary thyroid carcinoma depends on age. Thyroid 11:953-957
26. Dammann F, Horger M, Mueller-Berg M, Schlemmer H, Claussen CD, Hoffman J (2005) Rational diagnosis of squamous cell carcinoma of the head and neck region: comparative evaluation of $C T, M R I$, and 18 FDG PET. Am J Roentgenol 184:1326-1331

27. Mancuso AA, Harnsberger HR, Muraki AS, Stevens MH (1983) Computed tomography of cervical and retropharyngeal lymph nodes: normal anatomy, variants of normal, and applications in staging head and neck cancer. Part II: pathology. Radiology 148:715-723

28. Yousem DM, Som PM, Hackney DB, Schwaibold F, Hendrix RA (1992) Central nodal necrosis and extracapsular neoplastic spread in cervical lymph nodes: MR imaging versus $C T$. Radiology 182:753-759

29. King AD, Tse GM, Ahuja AT, Yuen EH, Vlantis AC, Te EW (2004) Necrosis in metastatic neck nodes: diagnostic accuracy of $C T$, MR imaging, and US. Radiology 230:720-726

\section{Publisher's Note}

Springer Nature remains neutral with regard to jurisdictional claims in published maps and institutional affiliations.

\section{Submit your manuscript to a SpringerOpen ${ }^{\circ}$ journal and benefit from:}

- Convenient online submission

- Rigorous peer review

- Open access: articles freely available online

- High visibility within the field

- Retaining the copyright to your article

Submit your next manuscript at $\boldsymbol{\nabla}$ springeropen.com 